\title{
Écologie des Cératopogonidés de la plaine d'Alsace.
}

\section{I. - Le genre Culicoides des sols humides du Ried}

\author{
par M. KREMER, J.-P. RIEB et C. REBHOLTZ \\ (collaboration technique : J.-C. Delecolle), \\ Institut de Parasitologie, Faculté de Médecine de Strasbourg, F 67000 Strasbourg.
}

\section{Résumé.}

Le Ried d'Alsace, ensemble de paysages humides de plaine, est présenté au point de vue géomorphologique, pédologique et botanioue. Quatorze espèces de Culicoides y sont étudiées au point de vue importance numérique, répartition topographique, biotope, densité par unité de surface et associations spécifiques. C. clastrieri, odibilis, cubitalis, pictipennis et musilator totalisent $98 \%$ des effectifs. Trois espèces halophiles, $C$. riethi, circumscriptus et salinarius y sont considérées comme de bons indicateurs de la pollution chlorurée de l'Ill.

\section{Summary.}

Ecology of the Ceratopogonids of the Alsace plain. I. The genus Culicoides from the humid soils of the Ried.

The Ried of Alsace, a humid landscape of plain, is presented on the geomorphological, pedological and botanical point of view. Fourteen species of Culicoides are studied following numeric importance, topographic distribution, biotop, density per soil surface unit, and specific associations. C. clastrieri, odibilis, cubitalis, pictipennis and musilator represent $98 \%$ of all. Three halophilic species, C. riethi, circumscriptus and salinarius are good indicators of salt pollution of the river Ill.

L'écosystème rhénan de la plaine d'Alsace représente un terrain d'étude remarquable, constitué de zones humides qui sont d'autant plus précieuses pour le biologiste qu'elles vont se modifier inexorablement sous l'influence de l'homme, dans le sens d'un assèchement et d'une banalisation du paysage.

Reçu le 8 août 1977. 
Les Cératopogonidés n'en ont été que peu étudiés, et si dès 1961, M. Kremer, C. Vermeil et J. Callot signalaient les effets de la pollution chlorurée sur la faune des Culicoides, une étude systématique n'avait pas encore été réalisée. Par ce travail, nous débutons une série d'études qui seront consacrées à la biologie des Cératopogonidés (des différents genres) et aux rapports existant entre eux et les divers biotopes rencontrés en plaine: chorologie des espèces, effets des facteurs autoécologiques sur les stades immatures et les imagos, et influence des travaux humains (drainages, travaux hydrauliques, mise en culture) et des pollutions sur les populations des diverses espèces.

\section{A. - Le Ried (1)}

\section{Définition et localisation géographique.}

Le Ried est l'un des paysages les plus caractéristiques de l'Alsace. Localisé à la région médiane de la plaine, il forme des zones humides à prairies marécageuses entrecoupées de bosquets et de forêts, et traversées par de nombreuses rivières, des ruisseaux et d'anciens fossés de régulation du niveau des eaux.

Le nom de « Ried vient du vieux terme allemand « Riet » qui signifie roseau, et qui a également été appliqué aux laîches (genre Carex) caractéristiques des milieux humides.

Au Nord-Ouest de Strasbourg s'étendent des Rieds fragmentaires dans les régions de La Wantzenau-Kilstett et de Sessenheim.

Les deux Rieds les plus importants sont situés dans la région centrale de la plaine (fig. 1) :

- le Ried Centre-Alsace ou ello-rhénan (dépendant de l'Ill et du Rhin). Le plus étendu en surface, il s'étire sur près de $50 \mathrm{~km}$ du Nord de Colmar au Sud de Sírasbourg, enserré entre l'Ill à l'Ouest et le Rhin à l'Est. Il est traversé par de nombreuses diffluences de l'Ill, et par des rivières, souvent d'origine phréatique, telles la Blind et la Zembs. La bordure orientale du Ried ello-rhénan en contact du Rhin, est couverte par la remarquable forêt rhénane (2);

- le Bruch de l'Andlau. «Bruch» en vieil allemand désigne des zones humides à aulnes. Le Bruch de l'Andlau est localisé au Nord-Ouest du Ried Centre-Alsace, et en est séparé par une bande de cultures où s'alignent de nombreux villages et où passe l'axe routier Strasbourg-Sélestat. Il s'adosse à l'Ouest au rebord des collines sous-vosgiennes. Ce Ried est traversé par de petites rivières qui descendent des vosges : l'Andlau et l'Ehn.

(1) Nous empruntons de nombreuses données à la remarquable étude réalisée par R. Carbiener (1969): Le Grand Ried d'Alsace. Ecologie d'un paysage.

(2) Nous prenons le terme de «Ried» dans son sens le plus large et nous englobons dans cette étude la forêt rhénane qui est, en fait, le prolongement du Ried sous sa forme la moins transformée par l'homme, et en même temps, l'expression la plus extrême de cet «écosystème rhénan ». 


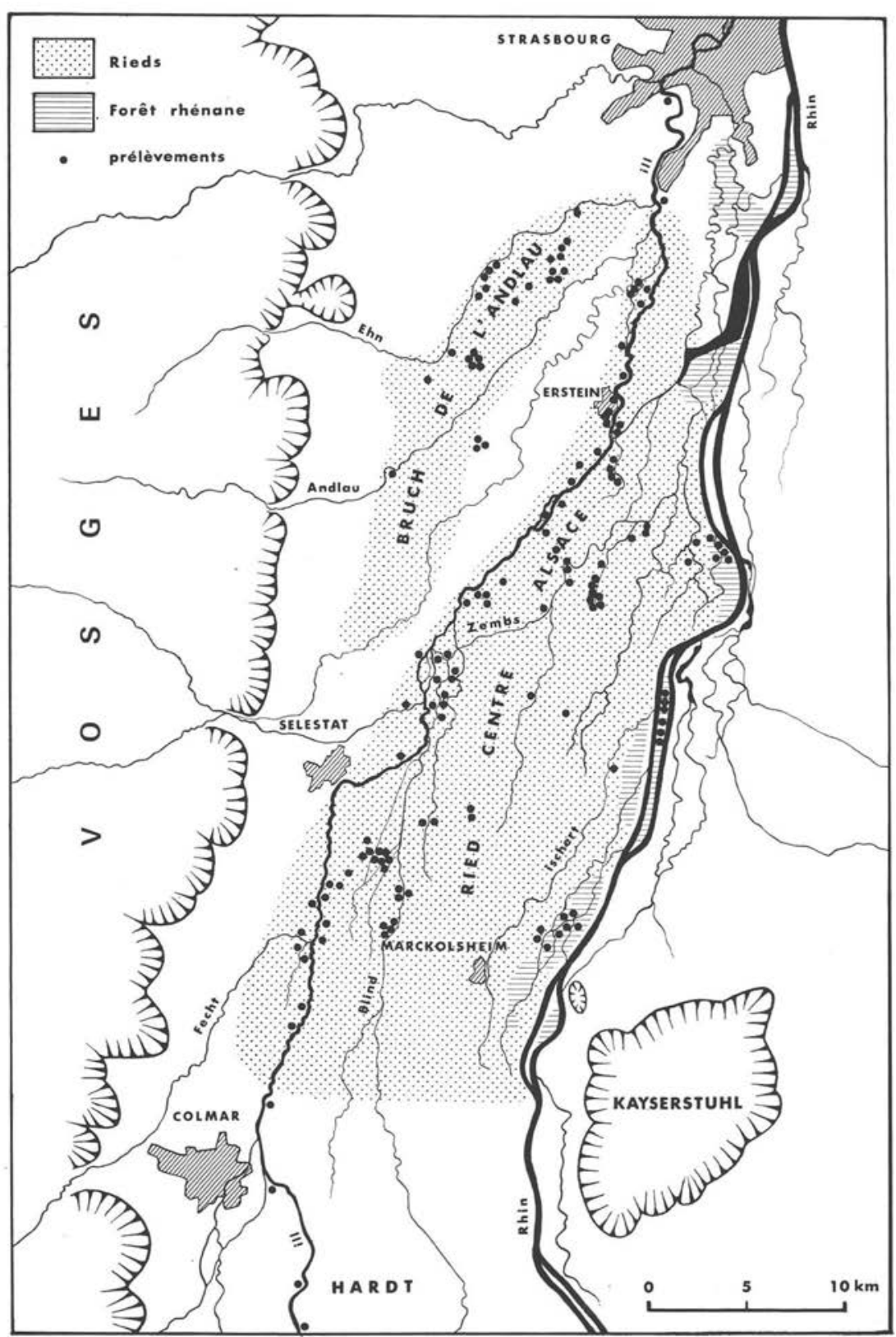

FIg. 1. - Situation géographique du Ried dans la région médiane de la plaine d'Alsace. 


\section{La formation du Ried.}

L'existence du Ried est une conséquence de l'épandage alluvionnaire du Rhin. A l'époque glaciaire, le Rhin — puissant torrent alpin — dépose dans le fossé tectonique alsacien un vaste cône de déjection à pente faible, très allongé dans le sens Sud-Nord, et légèrement bombé dans le sens transversal. Ce remplissage du couloir rhénan par des alluvions calcaires se fait sur plus de 200 mètres d'épaisseur (N. Théobald, 1948). Dans la région amont, se déposent les éléments les plus grossiers: graviers et galets de gros calibre formant le cône de déjection de la Hardt. La pente du Rhin, dans cette partie Sud de l'Alsace, est environ de $1 \%$. A la hauteur de Colmar, la pente du Rhin diminue $(0,7 \%$ ) et le fleuve dépose vers le Nord un glacis d'épandage formé d'alluvions plus fines: graviers, sables et limons qui s'interstratifient au hasard de nombreux chenaux instables.

Au post-glaciaire, le Rhin véhicule moins d'alluvions mais conserve un débit très important. Dans le secteur amont, au lieu de continuer à exhausser le cône de déjection, le fleuve au contraire, va creuser un lit dans ses propres alluvions. A la hauteur de Mulhouse, l'entaille atteint 15 mètres de profondeur.

A partir de la région de Colmar (entre Neuf Brisach et Marckolsheim) où la pente diminue, le Rhin perd sa puissance érosive: il ne creuse plus ses alluvions, mais les remanie. Le fleuve divague en un réseau de diffluences de type deltaïque, et dépose des levées sablo-limoneuses de rives qui ont tendance à rehausser légèrement le terrain bordant le lit majeur, le plus fréquemment inondé.

Cette mise en place géomorphologique conzitionne le tracé de l'Ill, la présence d'une nappe phréatique et ses phénomènes d'écoulement, et finalement l'existence des Rieds.

L'Ill venant des contreforts du Jura alsacien, butte contre le flanc Ouest du cône de la Hardt et est déviée vers le Nord. Dans la région moyenne de la plaine, le bombement transversal du glacis rhénan diminue, mais l'Ill est maintenue à distance du Rhin par les levées de rives de ce dernier. Ce n'est qu'au Nord de Strasbourg, après un cours de près de $120 \mathrm{~km}$, que l'Ill se jette dans le Rhin.

Les graviers rhénans formant le soubassement de la plaine sont imprégnés d'une importante nappe d'eau souterraine. Cette nappe phréatique est alimentée par les infiltrations des rivières vosgiennes dans leurs cônes et par les pertes de l'Ill dans son cours amont dans les cailloutis poreux de la Hardt. La nappe se déplace de quelques décimètres à quelques mètres par jour en direction du Nord-Est dans la région méridionale, puis vers le Nord. A la hauteur de Colmar — où la pente des alluvions rhénanes diminue - et dans la zone située plus au Nord, la nappe phréatique se rapproche de la surface, humecte les racines des végétaux et crée les paysages humides des Rieds. En certains points, la nappe affleure la surface du sol et forme des résurgences appelées «Donnerlöcher». L'eau sous pression perce la couche d'argile et de tourbe et apparaît au fond d'un cratère pouvant atteindre 2 mètres de profondeur ( Belle Source» du Ried de Herbsheim). Cette eau est cristalline, oxygénée (alluvions 
poreuses), sténotherme ( $10^{\circ} \mathrm{C}$ en hiver, $14^{\circ} \mathrm{C}$ en été). Elle s'écoule en surface en formant des rivières phréatiques ou «Brunnwasser», peuplées de truites.

Le niveau supérieur ou toit de la nappe phréatique est défini par le niveau du Rhin qui fait office de collecteur. Ainsi, dans le cône de la Hardt où le Rhin a creusé un lit de 15 mètres de profondeur, les eaux de la nappe s'infiltrent jusqu'à ce niveau, alors que dans la région moyenne de la plaine, le fleuve n'étant plus encaissé, la nappe répparaît à la surface.

Cette dépendance du niveau du fleuve a une conséquence écologique importante, car le Rhin étant un fleuve à régime alpin, il présente de fortes crues cycliques en été. Les variations du niveau du Rhin entraînent une oscillation verticale de la nappe qui fonctionne comme une pompe aspirante et foulante aérant le sol. Ces oscillations sont surtout sensibles idans la forêt rhénane bordant le fleuve, et dans le Ried attenant.

Ce phénomène, associé à la crue estivale qui procure aux plantes de l'eau en très grandes quantités au moment le plus chaud de l'année, explique le développement exubśrant de la forêt du Rhin qui prend un aspect subtropical tout à fait exceptionnel en Europe (plus de 50 espèces ligneuses, taille géante de certains arbres, lianes).

Par contre, les crues de l'Ill - rivière dépendant du climat océanique - ont lieu à la fin de l'hiver et inondent le Ried de l'Ill en y déposant des limons.

\section{Sols et végétations.}

En fonction de la géomorphologie et de la nature des sols, tous hydromorphes, R. Carbiener (1969) distingue plusieurs sous-unités pédologiques:

- le Ried noir, dépressions les plus marécageuses et les plus typiques du Ried dont le fond est en contact permanent de la nappe. Ce sont des sols noirs, riches en matières organiques où la minéralisation est inhibée par l'anaérobiose provoquée par l'excès d'eau. Il s'agit soit de tourbe calcique et alcaline (les ions calcium sont apportés par la nappe phréatique circulant dans les graviers rhénans calcaires) épaisse de 1 à 2 mètres, soit d'anmoor. Le sous-sol est un glei, argile vendâtre contenant du fer ferreux et ennoyé en permanence par la nappe.

- le Ried gris, localisé aux zones inondables de l'Ill. Le sol est un hydromull, humus minéralisé par les alternances dessication-ennoyage, et enrichi périodiquement par des argiles déposées lors des crues de l'Ill.

- le Ried brun, sols des levées-terrasses, plus secs car éloignés du niveau supérieur de la nappe. Ces sols bruns riches en calcaires et à matière organique fortement minéralisée sont mis en culture.

- le Ried blond, situé près du Rhin, est formé de sols très jeunes, alluvions sableuses et calcaires déposées par les crues à courant vif du Rhin.

La végétation originelle du Ried était forestière, à l'exception des bas-fonds les plus humides du Ried noir occupés par des Carex. Sur ces sols marécageux, la forêt caractéristique mais actuellement peu répandue, est l'aulnaie (Bruchwald). En terrain 
un peu plus sec, le frêne s'associe à l'aulne. L'aulnaie à frêne est également présente sous forme de forêts-galerie le long des rivières phréatiques et des diffluences de l'Ill. Dans les zones inondables par les eaux de l'Ill se développent les forêts riveraines ou ripisylves (Auwälder). Aux essences précédentes s'ajoutent le chêne pédonculé et le charme formant une forêt dense et sombre conditionnant une strate arbustive peu fournie et une strate herbacée à floraison vernale.

La forêt du Rhin, grâce à la convergence de plusieurs facteurs stimulants (microclimat chaud et humide en été, crue estivale du Rhin et balancement de la nappe phréatique, substrat calcaire, poreux et aéré), présente une richesse en espèces végétales et une complexité structurale exceptionnelles pour l'Europe. Les bas-fonds à chenaux inondés sont occupés par le saule blanc et le peuplier noir. Les levées sont caractérisées par le chêne pédonculé et l'orme réalisant une strate arborescente très haute mais claire, favorisant une strate arbustive dense extrêmement riche en espèces (nombreux arbustes à baies). Certains bras morts du Rhin, abandonnés par les eaux vives depuis la régularisation du fleuve, sont partiellement envahis par des roselières.

Les prairies, caractéristiques du paysage actuel du Ried, sont exploitées depuis des siècles comme prairies de fauche. Les plus intéressantes sont les prairies humides du Ried noir. Elles hébergent la flore primitive du Ried caractérisée par un petit carex, le schoin noircissant (Schoenus nigricans) et une graminée, la molinie (Molinia coerulea), ainsi que plusieurs espèces très rares dont les quelques stations sont pratiquement les seules de France. (G. Ochsenbein, 1969).

Dans le Ried brun, plus sec, la graminée dominante est le brome dressé (Bromus erectus) formant le Mesobrometum riche en espèces.

Le Ried gris régulièrement enrichi par le limon des crues, présente des prairies grasses à fromental (Arrhenatherum elatius) dont le rendement fourrager est très élevé.

\section{B. - Les Culicoides du Ried}

\section{Prospection des gîtes larvaires et technique de prélèvement.}

Pendant la période de mars à octobre 1976, nous avons prospecté des sols du Ried susceptibles d'être des gîtes larvaires de Cératopogonidés. Des prélèvements ont été effectués dans différents milieux: résurgences de la nappe phréatique, ruisseaux phréatiques, fossés de drainage, trous d'eau partiellement ombragés en prairie, trous d'eau en forêt, bras morts de l'Ill en prairie, bras morts en forêt rhénane, gravières, eaux peu profondes stagnant sur substrat de gravier et de vase, algues vertes filamenteuses flottantes, ruisseaux et rivières à divers degrés de pollution, - ainsi que dans les villages : rivières, fossés et caniveaux riches en eaux ménagères ou en purin, fumier, sol de porcherie. 
L'année 1976 a été caractérisée par des conditions climatiques particulièrement chaudes et sèches, aussi certains ruisseaux étaient-ils totalement à sec, ce qui réduisit les investigations dans certains secteurs. Par contre, les rivières en étiage ont laissé à découvert d'importants bancs de vase qui ont pu être prospectés.

La terre humide ou la vase est prélevée en général sur la berge au niveau supérieur de l'eau, là où le substrat humide vient en contact de l'air et forme un milieu amphibie. Chaque échantillon est un décapage de 500 à $600 \mathrm{~cm}^{2}$ de surface sur une profondeur de $1 \mathrm{à} 3 \mathrm{~cm}$, et correspond à un volume d'environ $750 \mathrm{~cm}^{3}$. Ces prélèvements sont conservés au laboratoire à température ambiante et l'éclosion des adultes est contrôlée quotidiennement, ainsi que nous l'avons déjà indiqué dans d'autres publications.

\section{Résultats.}

Les éclosions de Cératopogonidés issus des prélèvements de la saison 1976 ont duré sans interruption jusqu’à fin mars 1977.

Les prélèvements de matières organiques azotées (purin de fumier, excréments de porcs) n'ont pas donné d'éclosions. Les vases provenant de fossés et de caniveaux à eaux ménagères, ainsi que les vases putrides de rivières très fortement chargées en matières organiques (Ehn à Meistratzheim, canal de l'Ehn à Krautergersheim) contiennent de très nombreux Psychodidés (plusieurs centaines d'éclosions par prélèvement), mais aucun Cératopogonidé. Par contre, les deux familles peuvent être associées dans des gîtes moins pollués, les Psychodidés y étant alors beaucoup moins nombreux.

Sur 173 prélèvements, 110 ont donné éclosion à des Cératopogonidés (63,5\% de l'ensemble des prélèvements). Le genre Culicoides est présent dans 99 prélèvements, soit $57 \%$ des cas.

Ces éclosions ont donné 8000 Cératopogonidés dont 6300 Culicoides. Le genre Culicoides représente donc $79 \%$ de l'ensemble (3). 14 espèces de Culicoides ont été mises en évidence ; il s'agit dans l'ordre numérique décroissant de : C. clastrieri Callot, Kremer et Deduit 1962, C. odibilis Austen 1921, C. cubitalis Edwards 1939, C. pictipennis (Staeger) 1839, C. musilator Kremer et Callot 1961, C. riethi Kieffer 1914, C. punctatus (Meigen) 1804, C. circumscriptus Kieffer 1918, C. subfascipennis analis Kieffer 1925, C. pumilus Winnertz 1852, C. poperinghensis Goetghebuer 1953, C. salinarius Kieffer 1914, C. pulicaris Linné 1758, C. stigma (Meigen) 1818.

Les cinq premières espèces citées totalisent $98 \%$ des effectifs de Culicoides (tableau I).

(3) Les autres genres de Cératopogonidés récoltés feront l'objet d'une étude ultérieure. 
M. KREMER, J.-P. RIEB ET C. REBHOLTZ

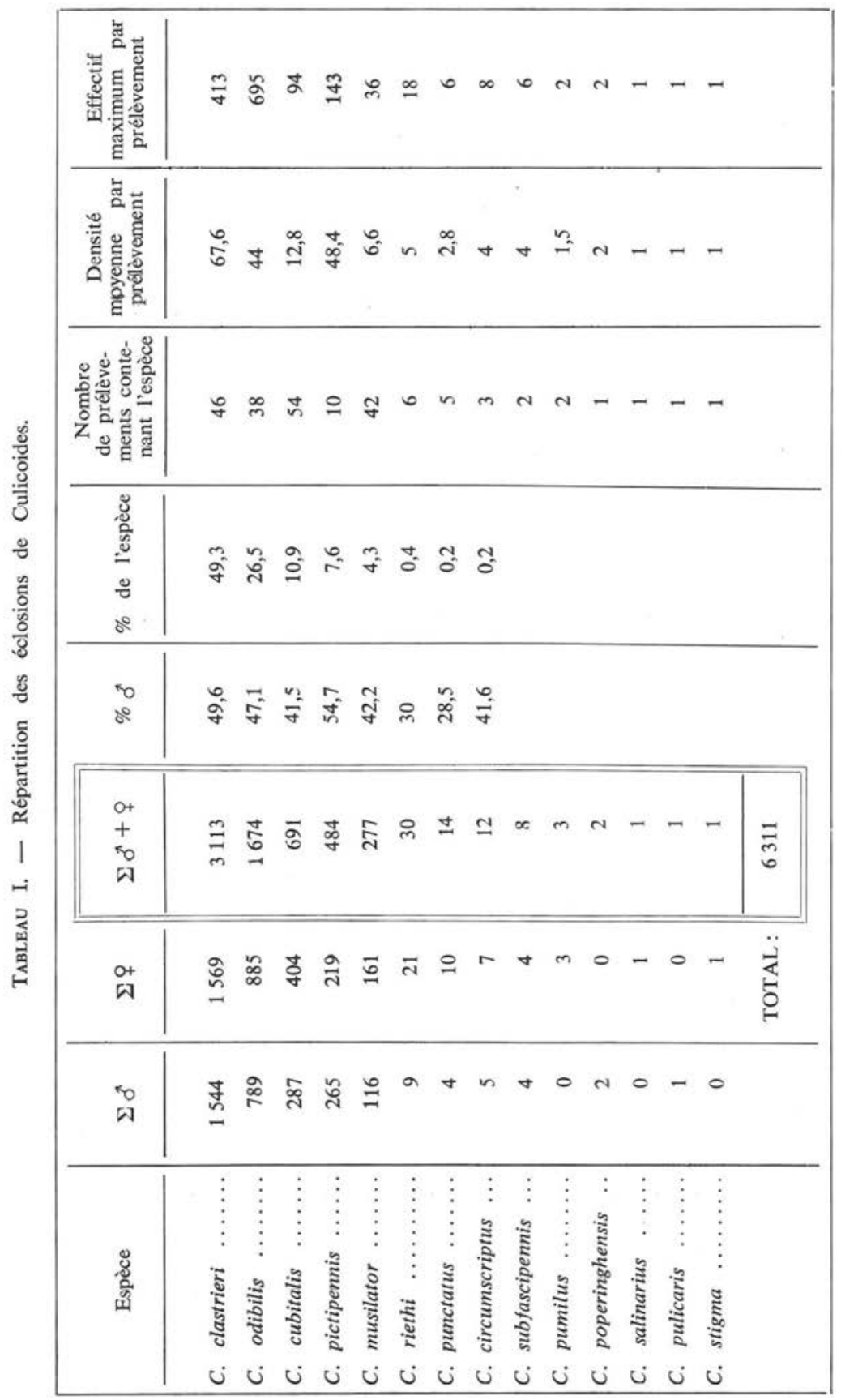


- C. Clastrieri $(49,3 \%$ des effectifs) est de loin l'espèce la plus fortement représentée en plaine. Au point de vue répartition topographique, elle vient en $2^{\circ}$ position (46\% des prélèvements positifs en Culicoides) et a été trouvée dans des biotopes de bords de rivières plus ou moins ombragées en lisière ou en sous-bois. Les substrats sont des bancs de vase légèrement émergés, vases de décomposition de feuilles d'arbres ou de plantes herbacées de la berge, débris végétaux en décomposition sur gravier, des vases sableuses, des sables fins, ou des terres plus fermes mais humides (Ried noir). L'espèce semble rechercher des eaux courantes et relativement limpides (rivières phréatiques) mais se trouve aussi en eaux moyennement chargées en matières organiques. Elle est plus rare en milieux stagnants. C. clastrieri a une grande tolérance thermique: nous l'avons trouvé dans les sables fins d'une résurgence où l'eau émergeait à $10,5^{\circ} \mathrm{C}$. La densité moyenne est la plus forte, avec 67,6 individus par prélèvement, soit 1130 individus $/ \mathrm{m}^{2}$. L'espèce est répandue le long des rivières phréatiques du Ried ombragées par leurs «forêts galeries» d'aulnes et de frênes, dans les forêts d'inondation de l'Ill et en forêt rhénane. C. clastrieri est souvent associé à odibilis, cubitalis et musilator, plus rarement à C. pictipennis, subfascipennis, poperinghensis, pulicaris et pumilus (fig. 3).

- C. odibILIs $(26,5 \%$ des effectifs) est une espèce bien représentée en plaine. Topographiquement moins répandue que $C$. clastrieri, elle vient en $4^{\circ}$ position avec une répartition de $38 \%$ des prélèvements positifs en Culicoides. $C$. odibilis se trouve parfois dans les mêmes biotopes à eau limpide que $C$. clastrieri, mais recherche de préférence des eaux plus chargées en matières organiques. On trouve $C$. odibilis dans des vases de bord de rivière ou d'eau stagnante, ombragées ou non, dans des trous d'eau en forêt et jusque dans les vases de l'Ill fortement polluée. C'est l'espèce dulçaquicole la plus tolérante aux milieux pollués; nous l'avons trouvée dans des secteurs de l'Ill chargés en chlorure de sodium, associée à des espèces halophiles dont nous parlerons plus bas. La densité moyenne est de 44 individus par prélèvement, soit 733 individus $/ \mathrm{m}^{2}$. Un prélèvement de vase effectué le 5 octobre 1976 dans l'Andlau, ruisseau pollué (eaux ménagères entre autres) à la sortie du village de Zellwiller, nous a révélé un peuplement mono-spécifique de $C$. odibilis remarquable par sa forte densité. Le prélèvement a donné naissance à 695 individus, ce qui correspond environ à une larve par $\mathrm{cm}^{2}$ de vase, soit 10000 larves par $\mathrm{m}^{2}$. Nielsen et Christensen (1975), étudiant des boues d'épandage d'eaux ménagères au Danemark, citent des densités de 200 larves $/ \mathrm{m}^{2}$ de $C$. odibilis, et de 10000 larves $/ \mathrm{m}^{2}$ de $C$. nubeculosus.

C. odibilis a été trouvé associé en gîtes d'eau douce à $C$. clastrieri, cubitalis, pictipennis, musilator, subfascipennis, punctatus, pulicaris et pumilus.

- C. cubitalis $(10,9 \%$ des effectifs) est l'espèce topographiquement la plus répandue ( $54 \%$ des prélèvements positifs en Culicoides). Sa densité est toujours relativement faible dans les prélèvements: 12,8 individus, soit 213 individus $/ \mathrm{m}^{2}$. C. cubitalis accompagne très souvent les deux espèces précédentes et se rencontre donc dans les gîtes précédemment décrits tout en évitant les gîtes les plus pollués occupés par C. odibilis. S'y ajoutent des gîtes de plein soleil tels que prairies marécageuses, canaux 
de drainage à terre ferme et argileuse, ou au contraire très ombragés tels que ruisseaux ou trous d'eau à eau stagnante et à substrat de feuilles en décomposition (forêt rhénane). Cet éclectisme fait que $C$. cubitalis peut se trouver associé à une ou plusieurs des 9 espèces suivantes: C. clastrieri, odibilis, musilator, pictipennis, subfascipennis, pumilus, punctatus, poperinghensis et pulicaris.

- C. PICTIPENNIS $\left(7,6 \%\right.$ des effectifs) vient en $5^{\circ}$ position quant à la répartition topographique (10\% des prélèvements positifs en Culicoides). Cette faible répartition s'explique par la spécificité de ses gîtes larvaires qui sont des fossés de drainage et des trous d'eau en prairie ou en sous-bois caractérisés par une présence temporaire de l'eau. La densité moyenne est relativement élevée : 48,4 individus par prélèvements soit 806 individus $/ \mathrm{m}^{2}$. Dans ces gîtes, C. pictipennis peut être associé à 6 espèces, mais qui sont présentes en quantités toujours très réduites; il s'agit de $C$. odibilis clastrieri, cubitalis, musilator, poperinghensis, punctatus.

- C. MUSilator est une espèce faiblement représentée au point de vue numérique $\left(4,3 \%\right.$ des effectifs) mais elle occupe la $3^{\circ}$ position quant à la répartition topographique $(42 \%$ des prélèvements positifs en Culicoides). C'est une espèce discrètement mais largement répandue ; elle peut être associée à 7 espèces de Culicoides : C. clastrieri, odibilis, cubitalis, pictipennis, punctatus, subfascipennis, pulicaris.

C. musilator se trouve dans les biotopes précédemment décrits, tout en recherchant de préférence des gîtes ombragés. Sa densité moyenne est faible : 6,6 individus par prélèvement soit 110 individıis $/ \mathrm{m}^{2}$.

Six autres espèces dulçaquicoles ont été trouvées en nombre très restreint :

- C. PUNCTATUS : 14 exemplaires provenant de 5 prélèvements. Ses gîtes larvaires sont constitués de vases de feuilles en décomposition en gîtes ombragés : bordure d'eau stagnante de bras morts en forêt rhénane ou dépressions humides dans certains bosquets du Ried. L'espèce a été trouvée associée à $C$. odibilis, cubitalis, pictipennis, musilator, pumilus.

- C. SUBFASCIPENNIS : 8 exemplaires provenant de 2 prélèvements. Les 2 gîtes sont des terres humides à débris végétaux avec eau stagnante en sous-bois, l'un en forêt de 1'Tlll, l'autre en forêt rhénane. Les espèces associées sont: C. cubitalis, clastrieri, odibilis, musilator.

- C. PumiLus : 3 exemplaires provenant de 2 prélèvements. L'un des gîtes est constitué de vase de feuilles en décomposition en bordure d'un bras mort rhénan en sous-bois, l'autre gîte est une terre argileuse dure d'une berge de ruisseau du Ried, sous un pont. Dans le premier gîte, $C$. pumilus est associé à $C$. odibilis et à $C$. punctatus, dans le deuxième gîte, l'espèce est associée à $C$. clastrieri et $C$. cubitalis.

- C. POPERINGHENSIS: 2 exemplaires provenant d'une boue d'un ruisseau du Ried, bordé de Carex, en sous-bois clair. Les espèces associées dans ce gîte sont C. cubitalis, clastrieri, pictipennis.

- C. pUlicaris : 1 exemplaire trouvé dans la boue noire en bordure d'une 
résurgence de la nappe phréatique dans le Ried noir, associé à $C$. odibilis, clastrieri, musilator, cubitalis.

- C. stigma : 1 exemplaire trouvé isolé dans la vase d'un bras de l'Ill à Ebersmünster, village du Ried au Nord de Sélestat. L'eau est polluée par les eaux ménagères et par le chlorure de sodium à une concentration voisine de $175 \mathrm{mg} \mathrm{Cl}-/$ litre.

Une prospection systématique des berges de l'Ill nous a permis de mettre en évidence l'existence de 3 espèces halophiles : C. riethi, circumscriptus, salinarius. Cette découverte est en relation avec la pollution chronique de l'Ill en chlorure de sodium sous l'effet des déversements massifs des mines du bassin potassique de Mulhouse. En effet, le chlorure de sodium non utilisé est déversé d'une part dans le Rhin, d'autre part dans l'Ill. L'étude de W. Krause et R. Carbiener (1975) montre des teneurs de chlorures dans l'eau de l'ull qui sont, de l'amont vers l'aval: 1000 à $500 \mathrm{mg} \mathrm{Cl}-/ 1$ entre Ensisheim et Illhaeusern, 250 à $175 \mathrm{mg} \mathrm{Cl}-/ 1$ entre Illhaeusern et Ebersmünster, 175 à $125 \mathrm{mg} \mathrm{Cl}-/ 1$ entre Ebersmünster et Sand, 125 à $95 \mathrm{mg} \mathrm{Cl}-/ 1$ entre Sand et Strasbourg (4). (Rappelons qu'une eau est considérée comme potable si, entre autres, elle contient moins de $70 \mathrm{mg} \mathrm{Cl}-/ 1)$. Nous avons trouvé les espèces halophiles dans l'ensemble de ces zones tout au long de l'Ill, mais en quantités relativement faibles.

- C. RIETHI : 30 exemplaires provenant de 6 prélèvements. Les gîtes larvaires sont des bancs de vase ou du sable, à surfaces couvertes d'algues vertes unicellulaires, de la région amont aux eaux brunes et nauséabondes contenant près de $1000 \mathrm{mg}$ $\mathrm{Cl}-/ 1$ (Bilzheim à $15 \mathrm{~km}$ au Sud de Colmar) jusqu'à Erstein dans la zone à $125 \mathrm{mg}$ $\mathrm{Cl}-/ 1$. Des 3 espèces halophiles, c'est l'espèce numériquement la plus importante et qui présente la plus grande extension le long de l'Ill: les prélèvements positifs se répartissent sur plus de $60 \mathrm{~km}$ de cours (fig. 2).

- C. CIRCUMSCRIPTUS: 12 exemplaires provenant de 3 prélèvements. L'espèce a été trouvée dans les mêmes gîtes que $C$. riethi dans les zones contenant de 1000 à $175 \mathrm{mg} \mathrm{Cl}-/ 1$ (de Bilzheim à Muttersholtz près de Sélestat).

- C. Salinarius: 1 exemplaire trouvé en association avec $C$. riethi et $C$. circumscriptus dans un grand banc de vase noire couverte d'algues vertes unicellulaires situé dans une boucle de l'Ill dans le Ried de Muttersholtz (zone à $175 \mathrm{mg} \mathrm{Cl}-/ 1$ ).

Un prélèvement effectué dans ce mème gîte en janvier 1977 a donné éclosion à un exemplaire de $C$. odibilis. D'autre part, $C$. riethi est présent dans l'Ill en amont d'Erstein et nous avons trouvé un gîte à $C$. odibilis $3 \mathrm{~km}$ en aval sur le même cours. Ces observations montrent la très grande tolérance de $C$. odibilis aux eaux salées (125 à $175 \mathrm{mg} \mathrm{Cl}-/ 1)$, l'espèce occupant par ailleurs les gîtes dulçaquicoles les plus variés. Le tableau des associations d'espèces rend compte de cet éclectisme de $C$. odibilis qui peut être associé à 8 espèces dulçaquicoles et 3 espèces halophiles (tableau II).

Le bassin potassique de Mulhouse a été prospecté dans la région de Wittelsheim par M. Kremer, C. Vermeil et J. Callot en 1961. Les auteurs ont trouvé en très gran-

(4) Tous les dosages de sel indiqués dans la suite du travail sont empruntés à Krause et Carbiener. Nous en avons contrôlé certains dans les vases et avons trouvé des concentrations très voisines. 
des quantités des larves de $C$. salinarius dans des eaux fortement salées contenant $13 \mathrm{~g}$. de chlorures par litre.

Les 3 espèces halophiles que nous avons trouvées le long de l'Ill dans des eaux peu salées, peuvent être considérées comme de bons bioindicateurs de pollutions salines, jusqu'à des taux de pollution relativement faibles.

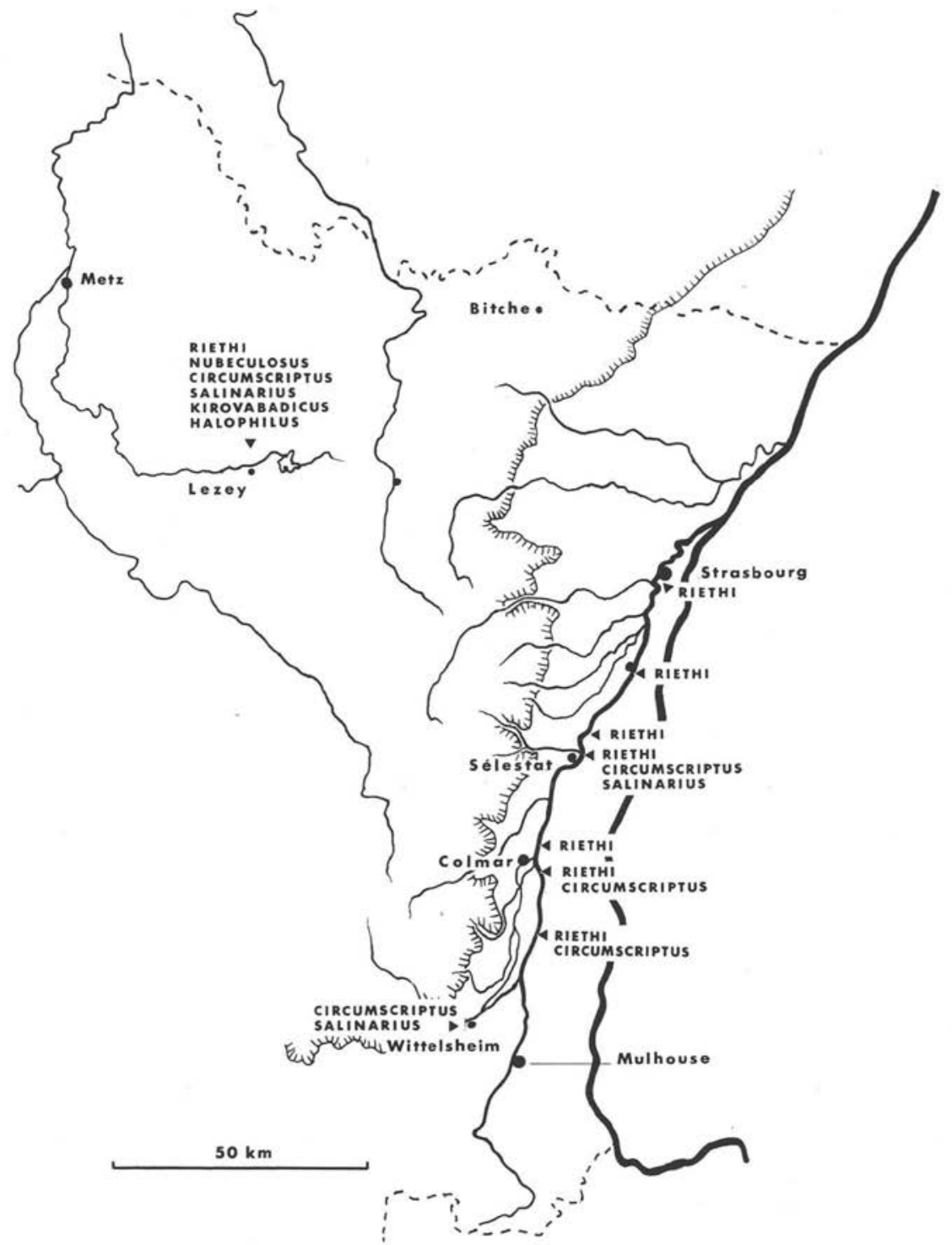

FIG. 2. - Répartition des espèces halophiles. 
L'exploitation de la potasse d'Alsace étant assez récente (1910), il se pose le problème de l'origine de ces espèces halophiles. Nous suggérons, à titre d'hypothèse, qu'elles pourraient provenir des salines de Lorraine où elles sont toutes présentes. Les Culicoides étant très mauvais voiliers, on peut penser à un transport des œufs ou des stades préimaginaux par les oiseaux aquatiques.

Tableau II. - Associations d'espèces.

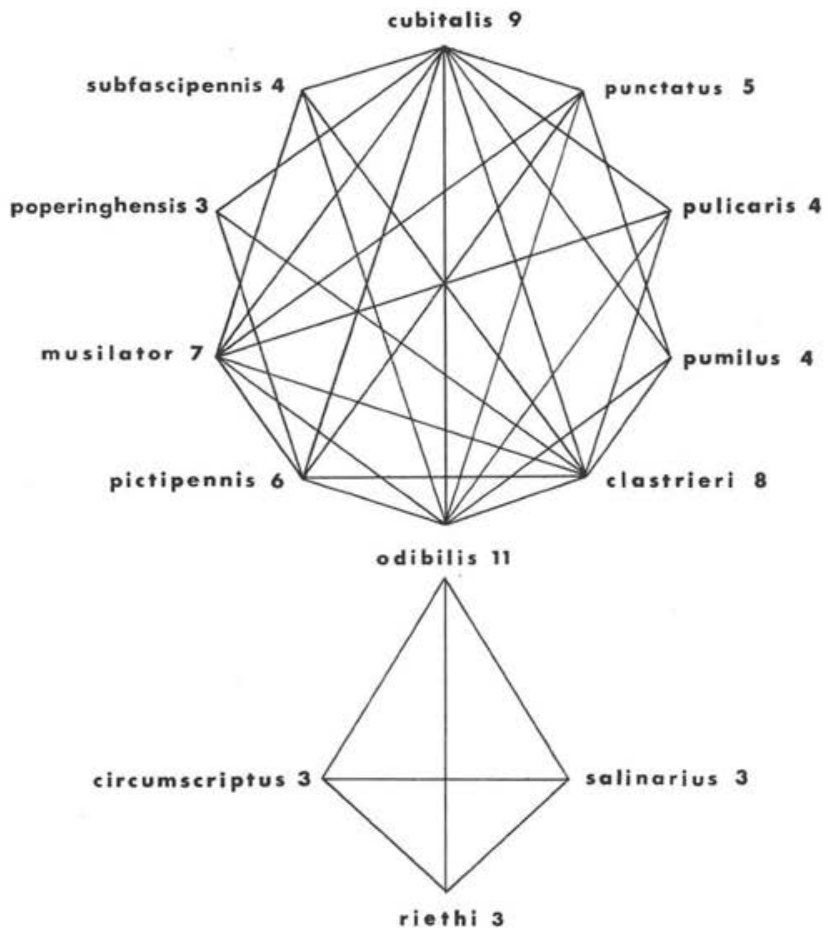

\section{III. - Discussion.}

Les espèces de Culicoides dulçaquicoles que nous avons pû étudier en plaine, ne semblent pas être liées à des biotopes spécifiques ou à certaines sous-unités pédologiques du Ried. Ces espèces sont, pour la plupart, largement tolérantes en ce qui concerne leur biotope larvaire, et colonisent tous les milieux aquatiques, quelle que soit la qualité des eaux. La seule constante de ces gîtes larvaires est un milieu humide semi-émergé. Ceci explique que plusieurs espèces peuvent être réunies dans un même gîte (jusqu'à 5 espèces par gîte). Dans le tableau d'associations d'espèces (tableau II), nous avons relié chaque espèce à toutes les autres espèces présentes avec elle dans différents prélèvements. Le chiffre suivant le nom d'espèce indique le nombre d'autres espèces qui lui sont associés. 
Cependant, l'association entre deux espèces n'implique pas obligatoirement une affinité identique ou réciproque de l'une des espèces pour le gîte de l'autre. Ainsi, par exemple $C$. pictipennis est associé à 6 autres espèces. $C$. pictipennis ayant un gîte larvaire spécifique, ne se trouvera pas dans les gîtes les plus fréquentés par ces 6 espèces ; mais ces dernières, plus éclectiques, coloniseront, entre autres gîtes, celui de C. pictipennis. Les espèces qui occupent indifféremment le plus de gîtes variés, sont celles qui sont associées au plus grand nombre d'autres espèces: C. odibilis (associé à 11 espèces), $C$. cubitalis (9), $C$. clastrieri (8), $C$. musilator (7). Le tableau montre d'autre part que $C$. odibilis, l'espèce la plus éclectique, fait quelques incursions dans les milieux faiblement salés et réalise un lien entre le groupe dulçaquicole et le groupe halophile.

Cette étude fait apparaître que $C$. clastrieri est l'espèce numériquement la plus répandue en plaine puisqu'elle représente la moitié des effectifs totaux. $C$. clastrieri est beaucoup moins représenté dans les Vosges où l'espèce ne compte que $5 \%$ de l'ensemble des Culicoides, dans la région de Haslach (J.-L. Geiss, 1973). Dans cette région, l'espèce largement dominante est $C$. cubitalis (79\% des effectifs); elle ne représente que $10,9 \%$ des effectifs en plaine où elle est cependant l'espèce topographiquement la plus répandue.

Les espèces de plaine qui n'ont pas été rencontrées dans les Vosges sont: C. musilator et $C$. stigma ainsi que, bien sûr, les espèces halophiles C. riethi, circumscriptus et salinarius.

Le sex-ratio des espèces numériquement significatives est normalement équilibré ; le pourcentage de mâles varie entre 41,5 et $54,7 \%$.

Plusieurs espèces découvertes en plaine dans les zones humides par J. Callot, M. Kremer et C. Paradis, en 1961 et 1962, n'ont pas été retrouvées. Il s'agit de C. jurensis, $C$. furcillatus, $C$. segnis, $C$. fascipennis. Les gîtes où ces espèces avaient été trouvées ont été détruits depuis.

\section{Bibliographie}

Callot (J.), Kremer (M.) et Paradis (C.), 1962. - Culicoides furcillatus n. sp. et Culicoides setosus Gutzevich, Diptères Cératopogonidés nouveaux pour la faune de France. Bull. Soc. Pathol. Exot., 55, 771-776.

Callot (J.), Kremer (M.), Geiss (J.-L.) et Hirtzel (C.), 1974. - Etude écologique de quelques Culicoides des Vosges (Diptères, Cératopogonidés). Ann. Parasitol. hum. comp., 49, 649-651.

Carbiener (R.), 1969. - Le Grand Ried d'Alsace. Ecologie d'un paysage. Bull. Soc. Indust. Mulhouse, 734, 15-44.

Carbiener (R.), 1970. - Un exemple de type forestier exceptionnel pour l'Europe occidentale: la forêt du lit majeur du Rhin au niveau du fossé rhénan. Vegetatio, 20, $97-148$. 
GeIss (J.-L.), 1973. - Etude documentaire sur la biologie du genre Culicoides Latreille (à propos d'espèces des forêts vosgiennes). Thèse médecine, Strasbourg, $197 \mathrm{p}$.

HIRTH (C.), 1974. - Eléments d'explication à la formation des Rieds ello-rhénans au Nord de Colmar. Bull. Soc. Hist. Nat. Colmar, 54, 21-44.

〈RAuse (W.) et CARbiener (R.), 1975. — Die Chloridkonzentration in den Gewässern der Oberrheinebene und ihrer Randgebirge. Erdkunde, Arch. Wiss. Geogr., 29, 267-277.

Kremer (M.), Vermeil (C.) et Callot (J.), 1961. - Sur quelques nématocères vulnérants des eaux salées continentales de l'Est de la France. Bull. Assoc. Philom. Als. Lorr., Strasbourg, 9, 1-7.

Kremer (M.), 1965. - Contribution à l'étude du genre Culicoides Latreille particulièrement en France. Lechevalier, édit., Paris, 295 p.

Marocke (R.), 1963-64. - Evolution des dépôts rhénans de la Haute-Alsace au cours du Pléistocène récent. Bull. Soc. Hist. Nat. Colmar, 51, 3-35.

Nielsen (B. O.) et Christensen (O.), 1975. - A mass attack by the biting midge Culicoides nubeculosus (Diptera, Ceratopogonidae) on grazing cattle in Denmark. A new aspect of sewage discharge. Nord. Vet. Med., 27, 365-372.

OChSENBeIN (G.), 1969. - Particularités botaniques des prairies naturelles du Ried. Bull. Soc. Indust. Mulhouse, 734, 45-50.

Théobald (N.), 1948. - Carte de la base des formations alluviales dans le Sud du fossé rhénan. Mém. $n^{\circ} 9$ du Serv. de la Carte Géolog. Alsace-Lorraine.

Ulrich (H.), 1963. - La Nature protégée. Dernières Nouvelles, Strasbourg, 209 p.

WALTER (J. M.), 1974. - Les dernières forêts alluviales rhénanes en Alsace-Bade. Bull. Ass. Philom. Als. Lorr. Strasbourg, 15, 101-112. 